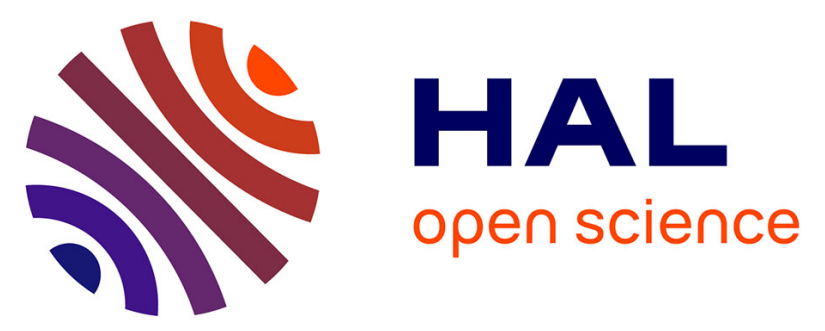

\title{
An improved acoustic method for the determination of the Boltzmann constant at LNE-INM/CNAM
}

Laurent E Pitre, Cécile Guianvarc'H, Fernando E Sparasci, Arnaud E Guillou, Daniel E Truong, Yves E Hermier, Marc E Himbert

\section{To cite this version:}

Laurent E Pitre, Cécile Guianvarc'H, Fernando E Sparasci, Arnaud E Guillou, Daniel E Truong, et al.. An improved acoustic method for the determination of the Boltzmann constant at LNE-INM/CNAM. Comptes Rendus. Physique, 2009, 10 (9), pp.835-848. 10.1016/j.crhy.2009.11.001 . hal-01512516

\author{
HAL Id: hal-01512516 \\ https://hal.science/hal-01512516
}

Submitted on 23 Apr 2017

HAL is a multi-disciplinary open access archive for the deposit and dissemination of scientific research documents, whether they are published or not. The documents may come from teaching and research institutions in France or abroad, or from public or private research centers.
L'archive ouverte pluridisciplinaire HAL, est destinée au dépôt et à la diffusion de documents scientifiques de niveau recherche, publiés ou non, émanant des établissements d'enseignement et de recherche français ou étrangers, des laboratoires publics ou privés. 


\title{
An improved acoustic method for the determination of the Boltzmann constant at LNE-INM/CNAM
}

\author{
Laurent Pitre, Cécile Guianvarc'h, Fernando Sparasci, Arnaud Guillou, Daniel Truong, \\ Yves Hermier, Marc E. Himbert \\ Laboratoire commun de métrologie LNE-CNAM, LNE-INM, Conservatoire national des arts et métiers, 61, rue du Landy, \\ 93210 La-Plaine-Saint-Denis, France
}

There is currently great interest in the international metrology community for new accurate determinations of the Boltzmann constant $k$, with a view to a new definition of the unit of thermodynamic temperature, the kelvin. Indeed, $k$ is related to the quantum of energy $k T$, where $T$ is the thermodynamic temperature.

The value of the Boltzmann constant can be obtained from measurements of the velocity of the sound in a noble gas. In the method described here, the experiment is performed in a closed spherical cavity. To obtain an accurate value for $k$, all the parameters of the experiment (gas purity, static pressure, temperature of the device, exact shape of the cavity, etc.) have to be carefully controlled. As correction terms have to be applied to the acoustic signals, the validity of the theoretical models from which they are derived is crucial.

The new determination carried out at the LNE-INM/CNAM is based on the same principles as in the acoustic experiment of Moldover et al. at NIST in 1988, which led to the most accurate determination of the Boltzmann constant up to now. However, several fundamental modifications and improvements have been made in this new experiment to measure and control the parameters that set the measured value of $k$.

Keywords: Metrology; Temperature; Boltzmann constant; Acoustics; Thermodynamics; Electromagnetism

Détermination de la constante de Boltzmann au LNE-INM/CNAM : une méthode acoustique de nouvelle génération. La détermination expérimentale de la constante de Boltzmann $k$ fait l'objet de plusieurs nouvelles expériences de haute exactitude au sein de la communauté des métrologues. En effet, reliée au quantum d'énergie d'agitation thermique $k T$, où $T$ est la température thermodynamique, la fixation de la valeur numérique de $k$ pourrait conduire à une nouvelle définition du kelvin, unité de température thermodynamique.

La mesure de la vitesse de propagation du son dans un gaz rare donne accès à la constante de Boltzmann. La présente expérience se propose d'effectuer de telles mesures dans une cavité résonnante fermée. Pour réaliser une mesure de haute exactitude il est indispensable de contrôler soigneusement et de mesurer de nombreux paramètres du dispositif expérimental (pureté du gaz, pression statique, homogénéité de température, forme géométrique de la cavité, etc.). La pertinence des modèles théoriques permettant de calculer les corrections à apporter à la situation nominale idéale de la propogation acoustique, et d'estimer l'incertitude, doit également être validée.

Les nouvelles mesures effectuées au LNE-INM/CNAM reprennent les principes de l'expérience acoustique réalisée au NIST en 1988 par Moldover et al., expérience ayant conduit, à ce jour, à la meilleure détermination expérimentale de $k$. La description 
des modifications et des améliorations apportées par le présent dispositif, les premiers éléments de caractérisation obtenus, et les bénéfices attendus en termes d'incertitude, font l'objet du présent article.

Mots-clés : Métrologie; Température; Constante de Boltzmann; Acoustique; Thermodynamique ; Électromagnétisme

\section{Introduction}

Since the 10th (1954) and 11th (1960) General Conference of Weights and Measures (CGPM), the definition of the unit of thermodynamic temperature has been based on a temperature fixed point, the triple point of water assigned the exact value of $273.16 \mathrm{~K}[1,2]$.

This definition links the magnitude of the unit of temperature, the kelvin, to a fundamental natural property of water, which is assumed to be uniform and sustainable. However, the practical realization of the unit is still based on a kind of artifact. One has to build cells where the triple point of water can be obtained, and indeed the precise international recommendations for doing this evolve over time.

In practice, the temperatures generated by different cells at the triple point of water are subject to various influences, the most important being:

- the effective pressure in the cell, which increases if the cell contains micro-cracks;

- the presence of impurities in the water due to an attack by the ultra-pure material (glass) cell component;

- the isotopic composition of water, which is difficult to measure precisely.

Thus, the uncertainty of the realisation of the triple point of water is unlikely to be reduced below the current value of $100 \mu \mathrm{K}$, which is higher than the differences in temperature that can be detected with the current sensors (of the order of a few microkelvins). This uncertainty has further to be propagated over the whole international temperature scale (ITS).

Moreover, the 20th and 21st CGPM strongly recommended the redefinition of the base units of the International System of Units (SI) in terms of fundamental constants of physics whose numerical values can be fixed [3,4], as has been done for the metre in 1983 [5]. In this context, the kelvin could be redefined by fixing the value of the Boltzmann constant $k$, linking the thermodynamic temperature to the quantum of thermal energy and to statistical thermodynamics. This would also have the advantage of being directly applicable to any primary measurement method of thermodynamic temperature, such as pyrometry. However, to obtain full benefit from such a new definition, it is firstly necessary to obtain:

- a sufficiently low relative uncertainty on the value of the Boltzmann constant, i.e. about $10^{-6}$;

- an international consensus on the numerical value.

\section{Acoustic method for the determination of the Boltzmann constant}

In a real gas, the velocity of the classical sound $c_{\mathrm{ac}}$ depends on the static pressure and can be written as follows:

$$
c_{\mathrm{ac}}^{2}=\gamma \frac{R T}{M}\left(1+\beta_{2}(T) P+\beta_{3}(T) P^{2}+\cdots\right)
$$

where $P$ is the static pressure, $T$ the thermodynamic temperature, $M$ the molar mass, $\gamma$ the ratio of heat capacities at pressure and constant volume, and $\beta_{i}$ are the coefficients of the virial expansion.

The expansion (1) shows that the velocity of sound depends only weakly on the static pressure in the gas. This is an advantage, as the measurement of the static pressure is currently impossible to obtain with a relative uncertainty lower than $1 \times 10^{-6}$. 
Extrapolating expansion (1) to zero static pressure ( $\gamma=5 / 3$ for a mono-atomic gas, $\left.R \equiv k N_{\mathrm{A}}\right)$ one obtains:

$$
\lim _{P \rightarrow 0} c_{\mathrm{ac}}^{2}=\frac{5}{3} \cdot \frac{k T}{m}
$$

where $N_{\mathrm{A}}$ is the Avogadro constant and $m=M / N_{\mathrm{A}}$ the atomic mass of the gas.

The velocity of sound in a gas can be determined experimentally by measuring the acoustic resonances in a perfect spherical cavity of known radius. It is related to the resonance frequencies $f_{\ln }^{\text {ac}} 0$ and the eigen-values for the wavenumber $k_{l n}^{\text {ac }}$ in the acoustic cavity. In the particular case of a spherical cavity of radius $a$, it is given by:

$$
c_{\mathrm{ac}}=\frac{2 \pi f_{l n}^{\mathrm{ac} 0}}{k_{l n}^{\mathrm{ac}}}=\frac{f_{l n}^{\mathrm{ac} 0}}{z_{l n}^{\mathrm{ac}}} 2 \pi a
$$

where $z_{l n}^{\mathrm{ac}}=k_{l n}^{\mathrm{ac}} a$ is the $n$th root of $j_{l}^{\prime}(z)=0$, with $j_{l}(z)$ the Bessel function of order $l$.

In making use of (3) in Eq. (2) and taking an average (written \langle\rangle ) over the different modes $l n, k$ comes to:

$$
k=\left\langle\frac{3}{5} \frac{m}{T}\left(\frac{2 \pi a}{z_{l n}^{\mathrm{ac}}}\right)^{2} \lim _{p \rightarrow 0}\left(f_{l n}^{\mathrm{ac} 0}\right)^{2}\right\rangle
$$

This expression is the basis of the present determination of the Boltzmann constant, deduced from the measurement of the frequencies of the acoustic resonances, extrapolated to zero static pressure, in a spherical cavity of measured radius, filled with a gas whose temperature is measured and whose atomic mass is known.

Expression (4) involving the acoustic resonance frequencies $f_{l n}^{\text {ac0 }}$ is valid only assuming that the conditions of propagation of the sound are "ideal" (a perfectly spherical cavity, a non-dissipative gas, uniform and perfectly reflecting walls, etc.). In practice, corrections have to be introduced to model the deviations from these ideal conditions. Quoting these corrections $\Delta f_{l n}^{\text {ac }}$ for each $f_{l n}^{\text {ac }}$, Eq. (4) becomes:

$$
k=\left\langle\frac{3}{5} \frac{m}{T}\left(\frac{2 \pi a}{z_{l n}^{\mathrm{ac}}}\right)^{2} \lim _{p \rightarrow 0}\left(f_{l n}^{\mathrm{ac}}+\Delta f_{l n}^{\mathrm{ac}}\right)^{2}\right\rangle
$$

Four major contributions to the uncertainty in the determination of $k$ can be highlighted from (5):

- the confidence in the models giving the corrections $\Delta f_{l n}^{\text {ac }}$ to be applied to take into account the non-ideal propagation of the acoustic waves;

- the accuracy in the determination of the volume (or the "mean radius") of the cavity;

- the uniformity and thermal stability of the whole resonator and in the gas;

- the knowledge of the molar mass of the gas.

The aim of the experiments accomplished so far in the different metrology laboratories to determine $k$ with an acoustic method has been to reduce the influence of these error sources, without increasing the effect of others or adding new ones. Our progress is described in the next section.

\section{Towards an improved performance at LNE-INM/CNAM}

The design of the experiment carried out at LNE-INM/CNAM has been optimised to reduce the impact of the error sources identified in Section 2. After a short insight on the design and properties of the experimental cavity (Section 3.1), the contribution to the uncertainty of these different error sources are discussed in Sections 3.2 to 3.5 and evaluated in Section 4.

\subsection{Practical choices}

To ensure the uniformity in temperature, the material chosen for the acoustic resonator is copper (highest thermal conductivity). It has also a high electrical conductivity (Section 3.3.2).

To benefit from the ability to obtain all thermophysical properties from ab initio calculations, the experimental gas is helium; furthermore, the atomic mass of helium has been determined accurately. 

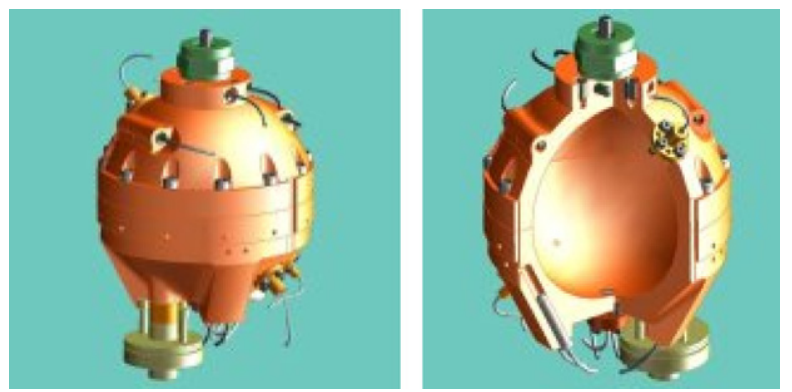

Fig. 1. Sketch of a quasi-spherical resonator designed at LNE-INM/CNAM.

The shape of the resonator is not perfectly spherical. As explained below, the volume of the cavity can be obtained by measuring the frequencies of the electromagnetic resonances, which is easier in the non-degenerate case. A quasispherical shape (practically a triaxial ellipsoid) is well suited as it also maintains values of the quality factors of the resonators as high as for a perfect spherical geometry.

\subsection{Acoustic model: Validation of the acoustic corrections}

\subsubsection{The acoustic pressure field and the resonance frequencies in the resonator}

An improved study of the existing models based on the fundamental equations of acoustics [6-8] has been completed in collaboration with the Laboratoire d'Acoustique de l'Université du Maine (LAUM UMR-CNRS 6613), France. It summarises in a single formalism all the various couplings and interactions resulting from the disturbances of the acoustic field in the practical cavity.

The acoustic field $p(\vec{r}, \omega)$ at point $\vec{r}$ (spherical coordinates $(r, \theta, \varphi))$ resulting from a source of pulsation $\omega$ must satisfy the equation of propagation inside the quasi-spherical volume $D$ and the continuity equations at the boundary surface $S$ (a non-uniform surface):

$$
\begin{aligned}
& \left(\Delta+k_{\mathrm{ac}}^{2}\right) p(\vec{r}, \omega)=0, \quad \vec{r} \in D \\
& \left(\frac{\partial}{\partial n}+j k_{\mathrm{ac}} \beta(\vec{r})\right) p(\vec{r}, \omega)=\sigma(\vec{r}), \quad \vec{r} \in S
\end{aligned}
$$

where $\Delta$ is the Laplace operator, $n$ the vector normal to the surface, $k_{\mathrm{ac}}$ the complex wave number which includes the viscous and the thermal effects in the volume $D, \beta(\vec{r})$ the surface specific admittance which describes the absorption and the reaction of the surface, and $\sigma(\vec{r})$ the effect of the acoustic source on the surface.

The system (6a), (6b) can be solved for the sound pressure function in the form of an expansion on the basis of spherical functions $\psi_{m}(\vec{r})$.

$$
p(\vec{r}, \omega)=\sum_{m} p_{m}(\omega) \psi_{m}(\vec{r})
$$

where the coefficients $p_{m}(\omega)$ in the expansion include all interacting disturbances of the acoustic field inside the resonator. One has to integrate over the almost spherical volume $D$ and to use Green's theorem to obtain the coefficients. The Neumann conditions at the boundary of the spherical field have also to be satisfied.

As a first step, this model can be applied to the simplest sphere already used: namely, the one used at the NIST for the previous determination of $k$ in 1988. To date, this resonator is the only one studied whose shape is the closest to a perfect sphere, the only significant discontinuities in the wall being related to the presence of the acoustic transducers (microphones). The corrective terms $\Delta f_{l n}^{\text {ac }}$ obtained from the model presented here are compared with the corrective terms computed twenty years ago in a way where coupling effects were neglected (Fig. 2).

Fig. 2 shows that the effect of coupling due to the perturbations of the field in the spherical cavity has only a small influence on the extrapolation to zero pressure. This seems to confirm the validity of the 1988 work on the calculation of the resonance frequencies in a sphere. This confirmation should also be verified for both models, by comparing the widths of the resonance curves obtained experimentally in 1988 with the predicted ones. It is, however, 


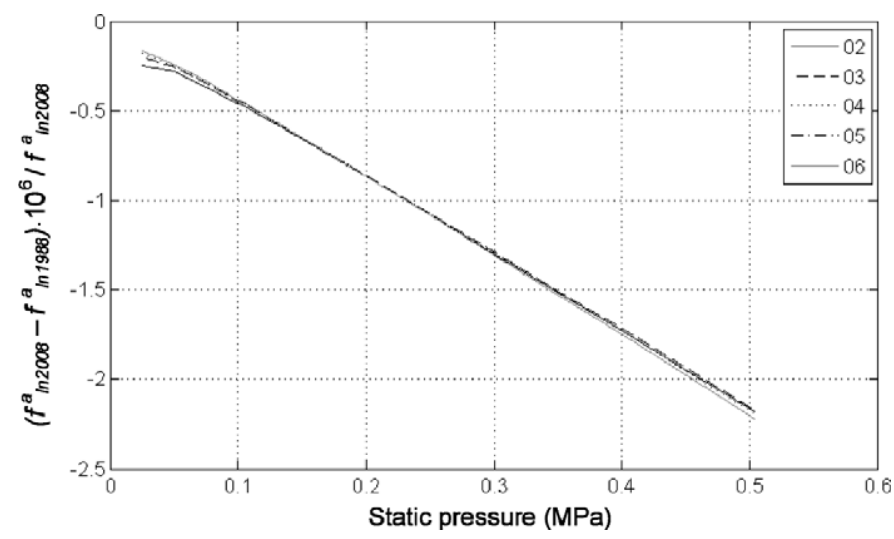

Fig. 2. Computed relative differences between the frequencies of acoustic resonances in a 'perfect' spherical cavity filled with argon at $T=273.16 \mathrm{~K}$ determined from the model presented here and the one used at NIST (1988), for the first five radial modes of the spherical cavity.

not excluded that the deviation from the perfect sphericity of the resonators used in LNE-INM/CNAM, coupled with other disturbances acting on the acoustic field, could lead to greater inter-linkages between modes. Further efforts are needed to ensure that the level of precision with which each of these disturbances is modelled is sufficient - given the low relative uncertainty expected. Some of these disturbances, especially the viscous and thermal effects which dominate in the acoustic resonator, should be estimated to one order higher than the previous one used.

Even if the intermodal couplings have only a small influence, in this case, on the acoustic resonance frequencies of the cavity, they have a significant influence on the spatial distribution as is exhibited in Fig. 3, which shows the maps of the acoustic pressure field. Indeed, exciting the cavity at the resonant frequency of a radial mode in the absence of intermodal coupling, the acoustic field should be uniform on the wall, which is not the case here.

It may be interesting to use this model to simulate and predict the shape of the acoustic field in different configurations (medium radius of the cavity, amplitude deviations from sphericity, position of acoustic electromagnetic transducers, tubes for the arrival and exit of the gas, etc.) to determine the optimal size and shape for the resonator and the optimal positions for the elements needed and positioned on the internal surface of the sphere. Thus, the analytical model could also provide assistance in the design and optimisation of a quasi-spherical resonator, soon to be undertaken for LNE-INM/CNAM.

A more complete view of this work can be found in [9].

\subsubsection{Resonance modes}

The acoustic field has to satisfy the boundary conditions for our non-ideal gas on non-ideal walls. Most of the induced dissipation occurs in the boundary layer of the gas. This effect results in a decrease in the quality factor of the cavity and in the broadening of the resonant curves. The observed width of the resonance curves have been compared to the expected ones, according to the simplest relevant dissipation model [10,11] (Fig. 4).

Computed values require the ab initio determination of some thermophysical properties of helium. For the different modes, extrapolation of the phenomenon to vanishing density result, without any adjustable parameter, in an accurate agreement between the experiment and the model. So the associate corrective terms can be accurately computed.

\subsubsection{The acoustic transducers}

The acoustic transducers used in the experiment are well characterised under atmospheric conditions. Their performance is not so well known in the specific conditions of pressure, of temperature and of the nature of the gas used in the present experiment. Studies (mostly experimental ones) have already been made in the past to determine the influence of the conditions of pressure and temperature, and of the composition of gas, on the characteristics of microphones [12,13]. Nevertheless, these studies cover a limited range of static pressure and gas mixture composition. Further studies (theoretical and experimental) have been undertaken in collaboration with INRIM to fix more precisely these points [14]. Preliminary results are shown in Fig. 5. 
(a) Mode 02 for $P=0.025 \mathrm{MPa}$

$$
|p(a, \theta, \varphi)| /\left|p_{\max }\right|
$$

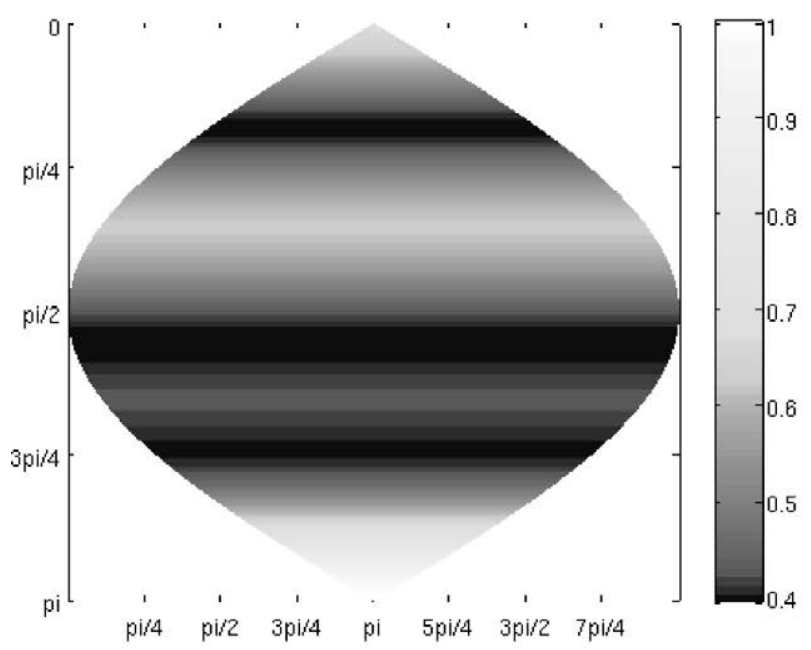

(b) Mode 05 for $P=0.5 \mathrm{MPa}|p(a, \theta, \varphi)| /\left|p_{\max }\right|$

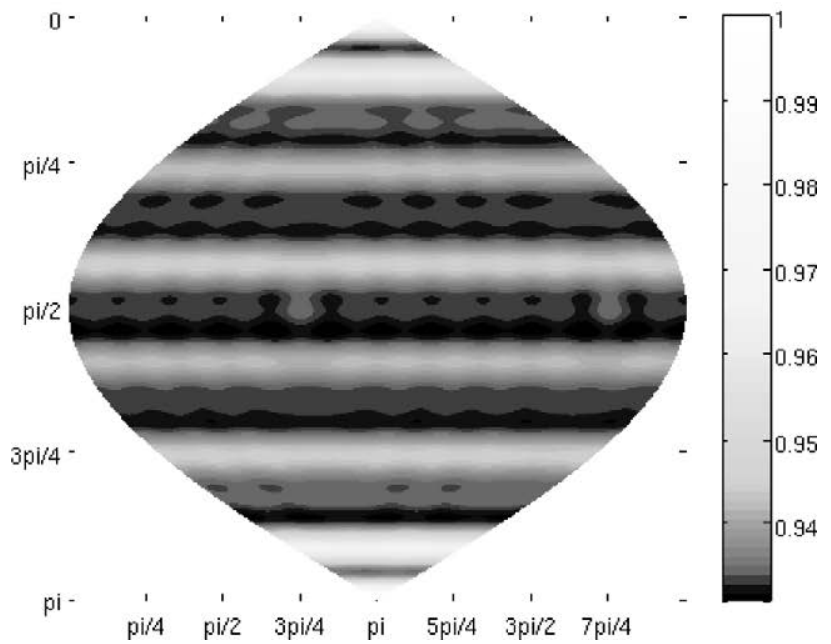

Fig. 3. Computed amplitude of the acoustic field, normalised at the source level, when the emitted signal is close to the harmonic resonance frequency of a given radial mode [mode 02 (a) and 05 (b), pressure level as quoted]. The source was supposed to be fixed at the south pole of the spherical cavity.

\subsection{Determination of the volume of the resonator}

To estimate the volume of the almost spherical resonator with a relative uncertainty better than $1 \times 10^{-6}$ is one of the major challenges of the experiment. It is not possible in our case to measure the volume by filling the resonator with a liquid material of known density and weighing the system, because mercury and copper are not well suited for this purpose. Consequently, two other methods have been used: direct three-dimensional (3D) mapping of the sphere, and measurement of the frequencies of the electromagnetic resonances of the metallic cavity.

\subsubsection{Coordinate Measurement Machine (CMM)}

The resonator is composed of two half-shells adjusted to form a quasi-spherical cavity (Section 3.1). The internal surfaces of the two hemispheres can be mapped by a method similar to that undertaken by May et al. [15]. Direct 


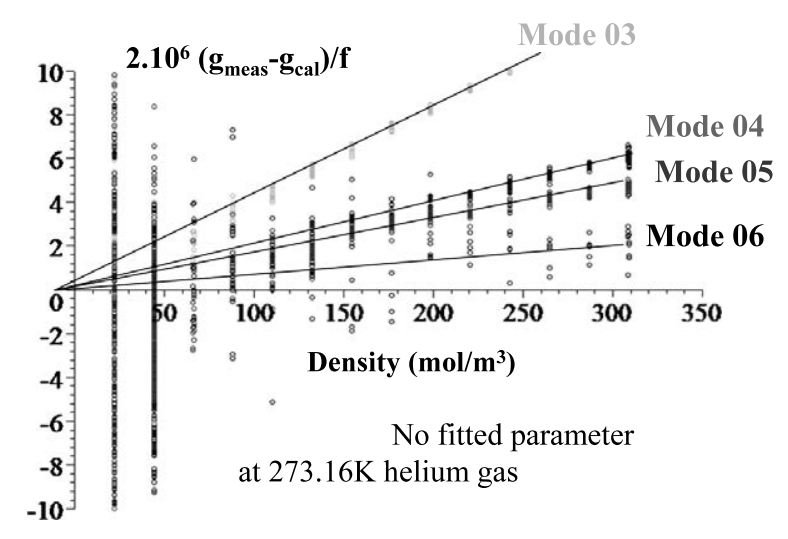

Fig. 4. Relative differences between the measured ( $\left.g_{\text {meas }}\right)$ and computed ( $\left.g_{\text {cal }}\right)$ half-width at half maximum (HWHM) of the resonances, versus the density in the gas. The different curves correspond to different modes, as quoted. Values are expressed in half parts in $10^{6}$ in order to be directly interpreted as the induced component of the uncertainty in the determination of $k$ (formula (5)).

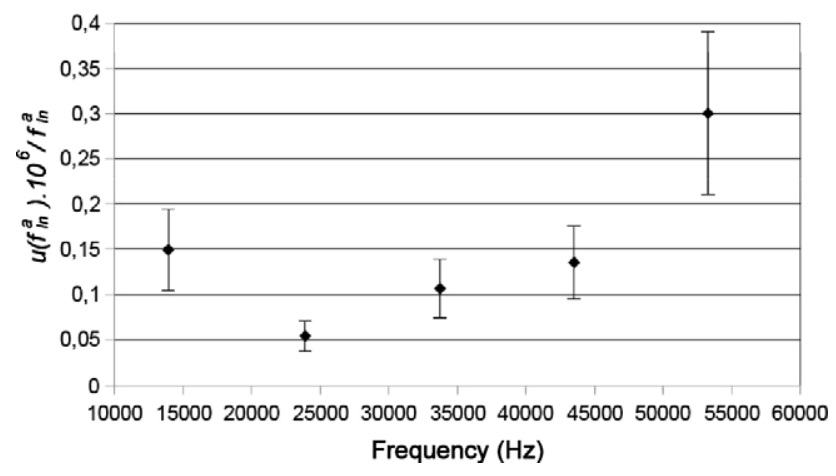

Fig. 5. Relative uncertainties (parts in $10^{6}$ ) related to the measurement of resonant frequencies of radial modes [02 to 06].

measurements of the temperature of the sphere during the mapping should also be made. The results of these threedimensional measurements, expressed in spherical coordinates $(r, \theta, \varphi)$ and the profile of the inner surface of the resonator can be mapped in terms of the spherical harmonic functions:

$$
r(\theta, \varphi)=a_{\mathrm{eq}}\left\lfloor 1-\sum_{l-1}^{\infty} \sum_{n-l}^{l} c_{l n} Y_{l n}(\theta, \varphi)\right\rfloor
$$

where $a_{\mathrm{eq}}$ is the radius of a sphere of equivalent volume and the coefficients are the coefficients $c_{l n}$ of the expansion on the basis of spherical harmonic functions $Y_{l n}(\theta, \varphi)$ [15]. Eq. (8) characterises the shape of the cavity compared to a perfect spherical shape.

This measurement method has already revealed defects in the form of a resonator made for LNE-INM/CNAM (Fig. 6).

However, it should be pointed out that with a three-dimensional mapping it is very difficult to determine the shape and exact volume of the resonator when it is assembled, because the static forces exerted during the assembly of the shells under the conditions of pressure and temperature of the experience are not completely known. Another method of measuring the volume, based on the measurement of electromagnetic resonance frequencies and described below, is therefore simultaneously used.

\subsubsection{Measuring the frequencies of the electromagnetic resonances}

In a perfectly spherical cavity with walls of infinite electrical conductivity, the resonant frequencies $f_{l n}^{\mathrm{em} 0}$ in vacuum of the different resonant modes of this electromagnetic "ideal" cavity depend on the radius $a$, on the speed of light 

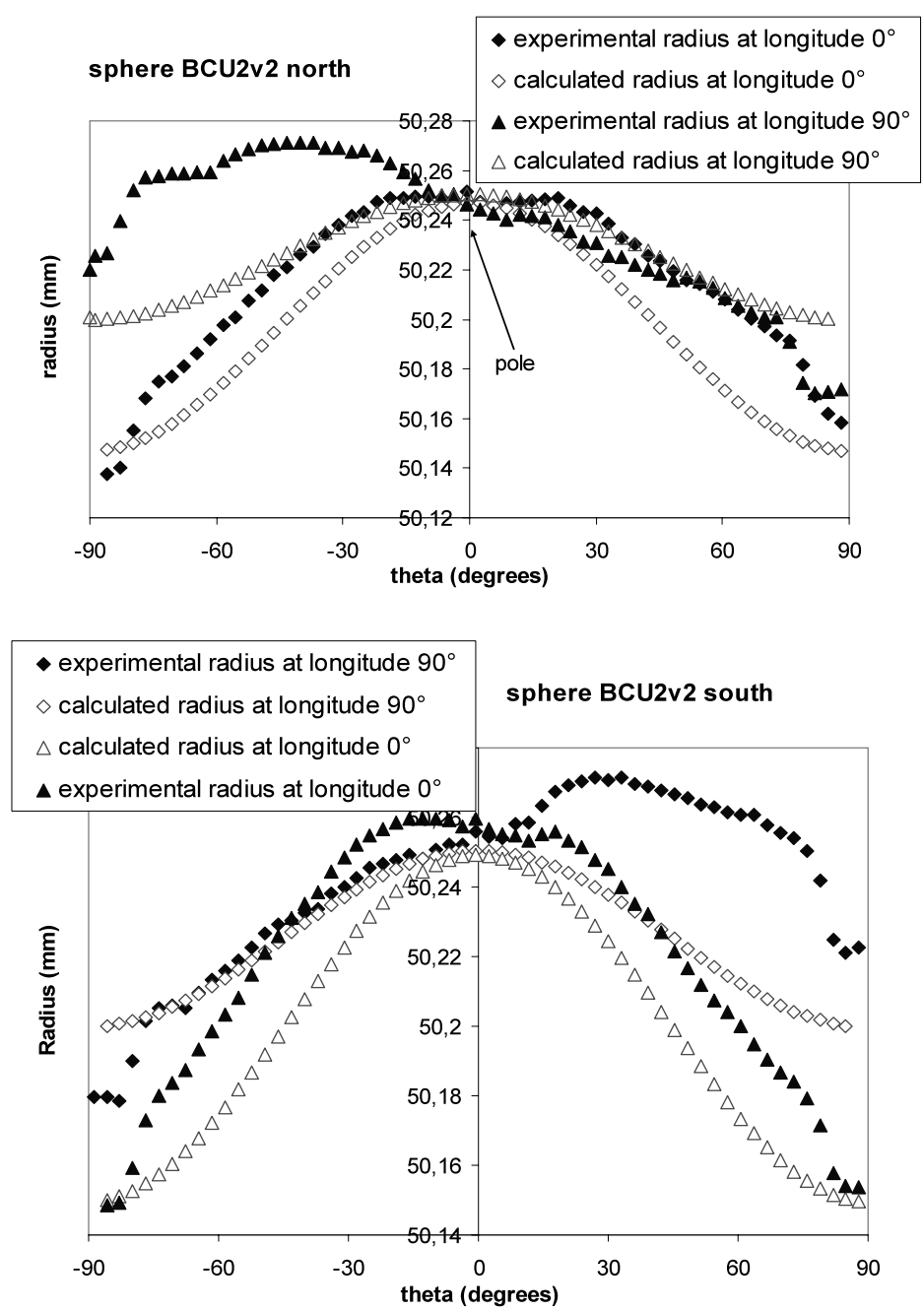

Fig. 6. Three-dimensional mapping of two sections of the acoustic resonator. The two curves correspond to the north and south hemispheres. The measured radius is plotted versus the latitude.

$c_{0}$ and on $z_{l n}^{\mathrm{em}}$, the $n$th root of the following equation involving the Bessel functions: $j_{l}(z)-z j_{l}^{\prime}(z)=0$ or $j_{l}(z)=0$, depending whether one observes a TM (transverse electric) or TE (transverse magnetic) mode. Hence, one can write:

$$
a=\frac{c_{0}}{2 \pi} \frac{z_{l n}^{\mathrm{em}}}{f_{l n}^{\mathrm{em} 0}}
$$

It is therefore possible, in principle, to determine accurately the radius of a spherical cavity by measuring the frequencies of the electromagnetic resonances. However, in practice, the cavity is not perfectly spherical. These defects create biases in the frequencies of electromagnetic resonance modes, which should be several times degenerate. Thus, it was deliberately chosen to use in the experiment a resonator with slightly predictable defects in the symmetry, to raise the degeneracy of the electromagnetic modes. This trick lowers the relative uncertainty on the measurement of the frequency of an electromagnetic resonance down to $10^{-9}$. The effective cavity, distorted in two directions, is a triaxial ellipsoid depending on two distortion parameters $\varepsilon_{1}$ and $\varepsilon_{2}$, according to, in Cartesian coordinates $(x, y, z)$, the equation:

$$
\frac{x^{2}}{\left(1+\varepsilon_{2}\right)^{2}}+y^{2}+\frac{z^{2}}{\left(1+\varepsilon_{1}\right)^{2}}=\frac{a^{2}}{\left(1+\varepsilon_{1}\right)^{2}\left(1+\varepsilon_{2}\right)^{2}}
$$




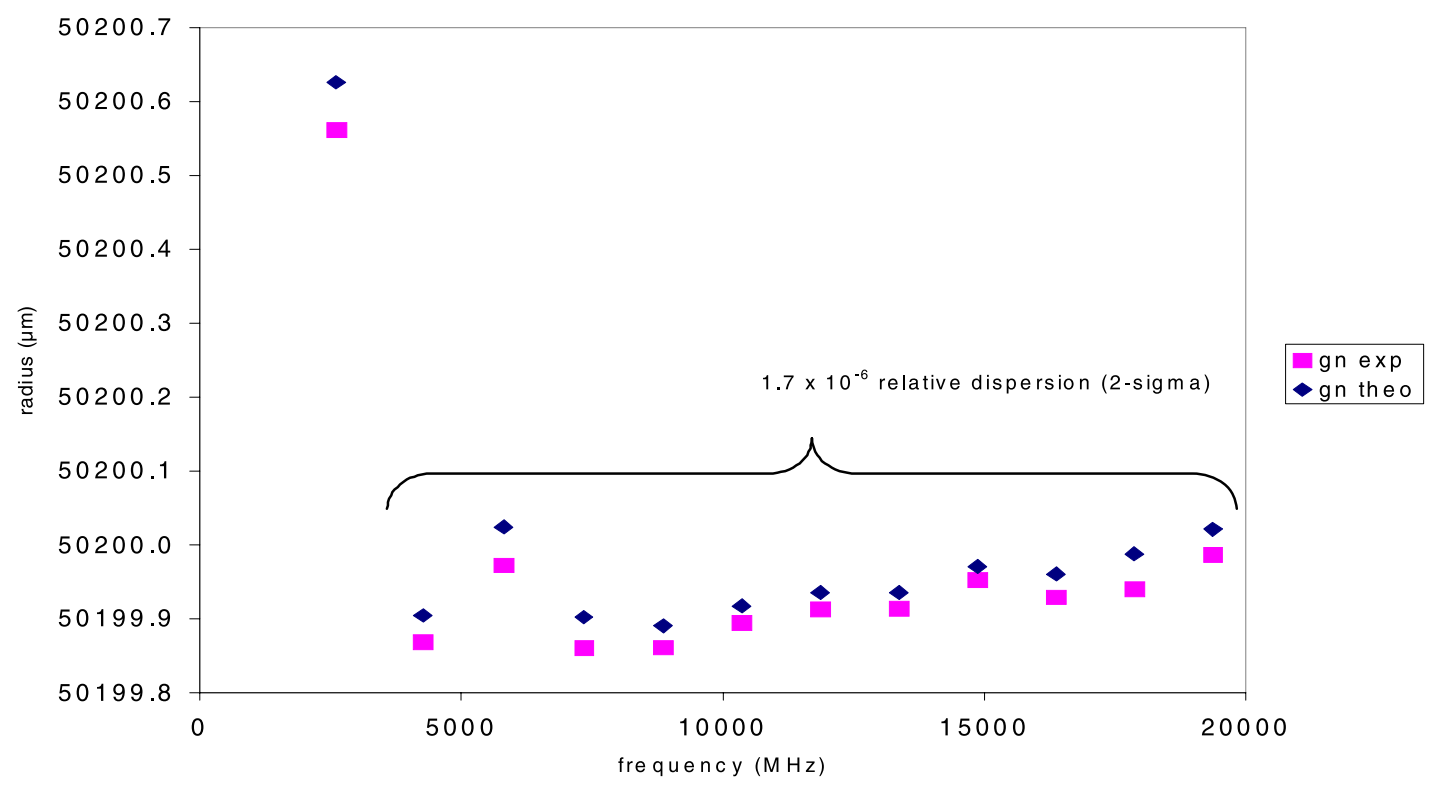

Fig. 7. Equivalent radius of the quasi-sphere BCU2, currently used by the LNE-INM/CNAM, obtained from the first 6 modes TM1, $n$ and the first 6 modes TE1, $n$. Microphone and capillary tubes are present on the surface. "theo" refers to the use of a tabulated value of the conductivity, whereas "exp" refers to an experimental evaluation issued from the observed width of the resonance curve.

The differences from the "ideal" propagation conditions (sphericity anisotropy, presence of acoustic transducers on the surface, finite electrical conductivity of the walls) require, as well as for acoustic measurements, the evaluation of some corrective terms $\Delta f_{l n}^{\mathrm{em}}$ to the measured frequencies $f_{l n}^{\mathrm{em}}$. The corrections should be derived from the proper modelling of the electromagnetic fields [16]. Thus, Eq. (9) turns into Eq. (11), where the equivalent radius $a_{\text {eq }}$ is the radius of a sphere having the same volume as the resonator, filled with helium gas of static pressure $P$ and temperature $T[15,19]$ :

$$
a_{\mathrm{eq}}=\frac{c_{0}}{2 \pi \sqrt{\varepsilon_{\mathrm{He}}(P, T)}} \cdot \frac{z_{l n}^{\mathrm{em}}}{\left\langle f_{l n}^{\mathrm{em}}+\Delta f_{l n}^{\mathrm{em}}\right\rangle}
$$

$\varepsilon_{\mathrm{He}}$ is the electric permittivity of helium.

With Eq. (5), one gets

$$
k=\left\langle\frac{3}{5} \frac{m c_{0}^{2}}{T}\left(\frac{z_{l n}^{\mathrm{em}}}{z_{l n}^{\mathrm{ac}}}\right)^{2} \lim _{p \rightarrow 0}\left(\frac{f_{l n}^{\mathrm{ac}}+\Delta f_{l n}^{\mathrm{ac}}}{f_{l n}^{\mathrm{em}}+\Delta f_{l n}^{\mathrm{em}}}\right)^{2}\right\rangle
$$

May and co-workers [15] have computed, to first order, the link between small distortions between the frequencies of the electromagnetic resonances in a quasi-spherical cavity, the radius $a_{\text {eq }}$ and the coefficients $c_{l n}$ (Eq. (8)). However, it appears that such a development is not sufficient, according to the actual distortion of the cavity used here and to the level of accuracy required. An expansion to order 2 has been recently developed by Mehl in close collaboration with our group in LNE-INM/CNAM [18]. The corrective term derived from this model, combined with the experimentally determined resonance frequencies for different electromagnetic modes, leads to values of the equivalent radius of the cavity (Fig. 7). The values have been appreciated in two different ways: using a reference value for the copper wall conductivity, or deducing the conductivity from the shape of the electromagnetic resonance curve. The results obtained for the different modes exhibit a high consistency; they do not differ by more than $0.2 \mu \mathrm{m}$, except for the first mode under consideration (the EM field is higher close to the wall). Furthermore the difference between the "theoretical" and the experimental evaluations of the radius for each given mode lies below the dispersion among the modes, which means that it will result in a negligible component of the uncertainty.

As a preliminary conclusion, the results of the measurement of the volume obtained with both methods show a good consistency. However, the relative uncertainty of the volume obtained by the three-dimensional mapping $\left(17 \times 10^{-6}\right)$ 


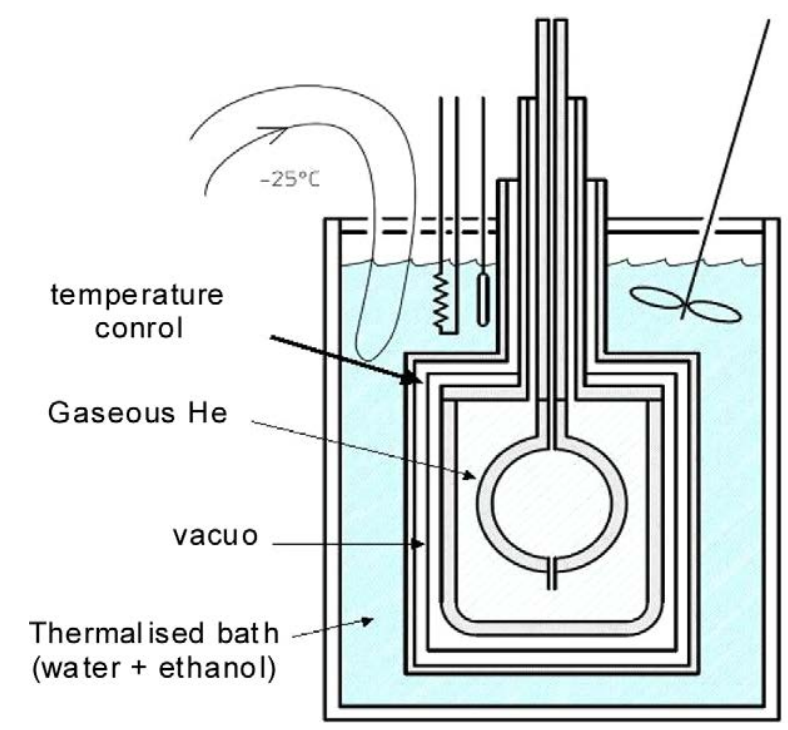

Fig. 8. Diagram of thermostat used at the LNE-INM/CNAM.

is much higher than that of the one obtained by the electromagnetic measurement $\left(0.85 \times 10^{-6}\right)$. It would therefore be appropriate to use a more accurate CMM to refine these results and compare more efficiently both methods.

\subsection{Uniformity and thermal stability}

\subsubsection{The thermostat}

The copper acoustic resonator is placed in a thermostat (Fig. 8) stabilising the temperature with a drift smaller than $0.1 \mathrm{mK} / \mathrm{h} \mathrm{[19]}$ and a thermal uniformity of better than $50 \mu \mathrm{K}$ is expected. While copper has a larger mechanical elasticity than stainless steel [16] the lack of the reproducibility of the mechanical elasticity is not likely to be a limiting factor in this project.

The temperature mapping of the resonator is performed by means of several capsule-type thermometers, directly calibrated at the triple point of water. However, the experiments described here are planned over several years and achieving a relative stability of the thermometers lower than $10^{-6}$ over a year is a real challenge. It has been necessary therefore to include in the measuring device a system to regularly re-recalibrate in situ all thermometers.

\subsubsection{Metallic cell for the triple point of water}

Glass cells currently used for the realisation of the triple point of water do not allow the quantitative monitoring of the evolution of the temperature as a function of the fraction of water in the different phases, especially the fraction of melted ice. Indeed, conventional glass cells are not designed to calibrate this type of thermometer and require adjustment of the temperature which can damage or degrade the measurement uncertainty. In the 1980s, the LNEINM/CNAM developed metallic copper cells for the triple point of water, and prototypes were calibrated at that time. A new prototype was designed, built and completed at the end of 2006 (Fig. 9a), realising the triple point of water using adiabatic calorimetric techniques (the advance in the phase transition is controlled by sending discrete energy quanta). With this prototype one has access to the temperature according to the fraction of water which is liquid [17].

The cell is associated with a comparison block (Fig. 9b) which allows the simultaneous calibration of several capsule-type thermometers, unlike conventional glass cells which allow only one at a time.

The short-term reproducibility of calibrations performed so far is about $0.2 \mathrm{mK}$, mainly set by the characteristics of dispersion of the cryostat used for these measurements. In the medium term, the drift of the observed temperature of the phase transition, probably due to the presence of impurities in the water, is about $35 \mu \mathrm{K} / \mathrm{month}$ (Fig. 10), which remains satisfactory but will be improved during the construction of new metallic cells.

Ultimately, it is planned to establish such a cell inside the cavity (Fig. 1) to become a reference for the calibration of thermometers during the experiment. 


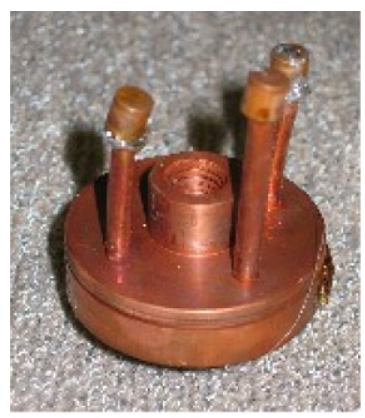

(a)

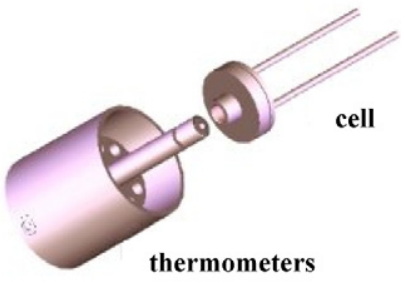

(b)

Fig. 9. (a) Prototype of a metallic cell (copper) at the triple point of water made at LNE-INM/CNAM; (b) Schematic of the cell block housing the thermometers.

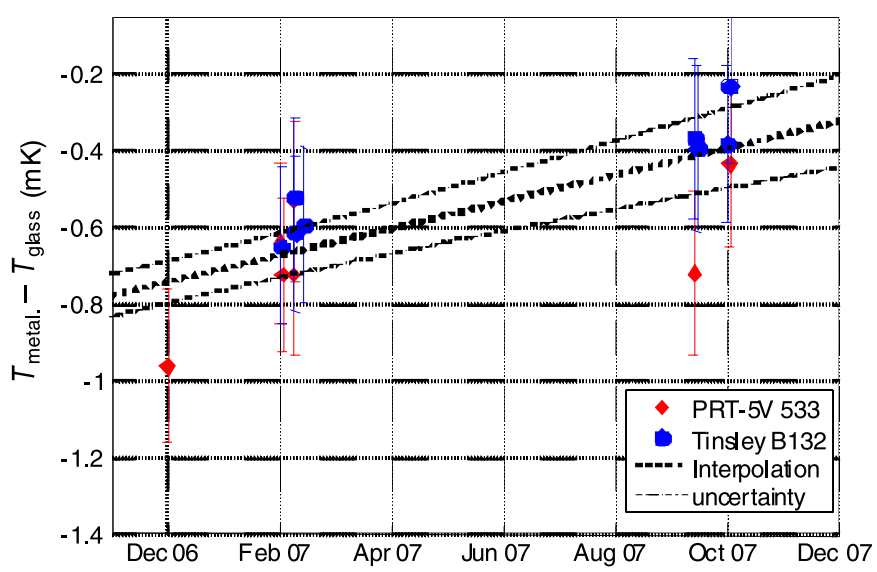

Fig. 10. Comparison of the results of temperature measurements obtained with a metallic cell and with a glass cell, at the temperature of the triple point of water.

\subsection{Determination of the molar mass of the gas}

The use of a light gas such as helium (here isotope 4) makes the results of the measurements of the velocity of sound very sensitive to the presence of impurities of higher mass. An enhanced control of the purity is therefore required. Thus, a continuous flow of pure helium is drawn through the sphere, thereby minimising the effects of the gas-desorption from the surface after pumping [16]. Pure gas flows out from a helium quality $6 \mathrm{~N}$ tank, passing through a trap at liquid helium temperature (Fig. 11), which condenses all the other gases with the exception of helium-3 (the hydrogen impurities are trapped by the getter). The relative concentration of helium-3 in helium- 4 remains less than $1.3 \times 10^{-6}$. Its effect on the velocity of sound is small and can be computed.

To validate the measurement of the molar mass of the gas in the resonator, the same experience will be carried out with purified argon. Comparisons of the velocities of sound will give access to the ratio of the molar masses $M_{\mathrm{He}} / M_{\mathrm{Ar}}$, which should scale as expected.

\section{Provisional uncertainty budget}

The partial uncertainty budget in Table 1 highlights the contribution of the improvements in the experience at LNE-INM/CNAM, compared to the previous experience at NIST:

- Even if the choice of helium instead of argon as a mono-isotopic gas complicates the experimental device, it allows a better determination of the molar mass; 


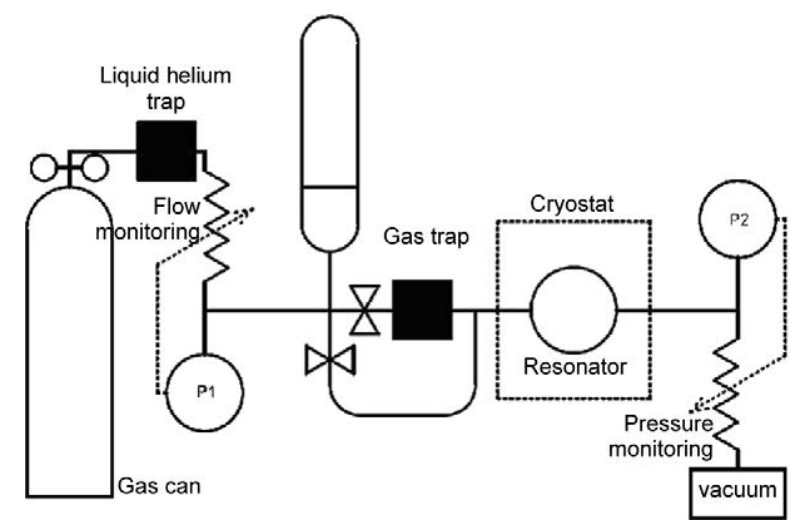

Fig. 11. Purification system of helium gas used in LNE-INM/CNAM.

Table 1

Assessment of uncertainties of the determination of $k$ (in parts in $10^{6}$, coverage factor equal to 1 ). $X$ represented the unknown uncertainty of the corrections due to the effect of the microwave antenna. n.d.: not yet computed.

\begin{tabular}{|c|c|c|c|}
\hline & $\begin{array}{l}\text { NIST } \\
1988\end{array}$ & $\begin{array}{l}\text { INM } \\
\text { sept. } 2007\end{array}$ & $\begin{array}{l}\text { INM } \\
\text { dec. } 2008\end{array}$ \\
\hline$(\text { Volume })^{2 / 3}$ & 0.80 & $\left(X^{2}+2.53^{2}\right)^{1 / 2}$ & 1.84 \\
\hline Temperature & 0.89 & 1.00 & 1.00 \\
\hline Molar mass & 0.80 & 0.24 & 0.24 \\
\hline Extrapolation $p \rightarrow 0\left(f_{n}+\Delta f_{n}\right)^{2}$ & 0.92 & 1.39 & 1.46 \\
\hline Microphone effect & n.d. & n.d. & negligible \\
\hline Reproducibility & n.d. & n.d. & 1.10 \\
\hline Combination & 1.7 & $>3$ & 2.7 \\
\hline
\end{tabular}

- The determination of the volume by measuring the resonance frequencies in the electromagnetic cavity gives consistent results for 11 different modes, which validates the second-order model developed by Mehl [18] and experimentally validated by us for a quasi-spherical cavity;

- The extrapolation to the value of the sound velocity at zero static pressure, obtained from the resonance frequencies of the acoustic cavity for 4 different radial modes, gives extremely close results.

In addition, other parameters have been introduced into the assessment of uncertainties in relation to NIST. The reproducibility of the measurement method is currently under study. Two measured isotherm curves were achieved with different purities of the helium gas and with different microphones. The relative difference between the determinations is $1.1 \times 10^{-6}$, and is presently being analysed.

In addition, a study of the effect of the acoustic transducers (microphones) in the cavity wall is currently underway. Another study has been performed to determine the effect of the microwave antenna on the determination of the volume of the cavity, poorly evaluated (illustrated by an $X$ in the balance sheet, Table 1) until recently. We can now use this appropriate model [24] of the electromagnetic field in the cavity taking into account the hole of the antenna to estimate the radius.

The extrapolation at zero pressure of the results already obtained along isotherms in 2007 and 2008 leads to the final results for $k$ in Fig. 12. The value found in this work is $k=(1.3806495 \pm 0.0000037) \times 10^{-23} \mathrm{~J} / \mathrm{K}$.

The analysis of the results shows that one of the main constraints of the acoustic method used here is the realisation of a quasi-spherical resonator with a precise shape (triaxial ellipsoid), adapted to the use of electromagnetic and acoustic models. So, it is planned to carry out measurements on a sphere of a nominal volume of $0.5 \mathrm{dm}^{3}$ whose shape is much closer to that expected. Finally, the analysis of the components of uncertainty shows that the uncertainty should be reduced by using a quasi-spherical resonator with a larger diameter. The use of such a tool should give access to a value of the Boltzmann constant with a relative uncertainty of $1 \times 10^{-6}$. 


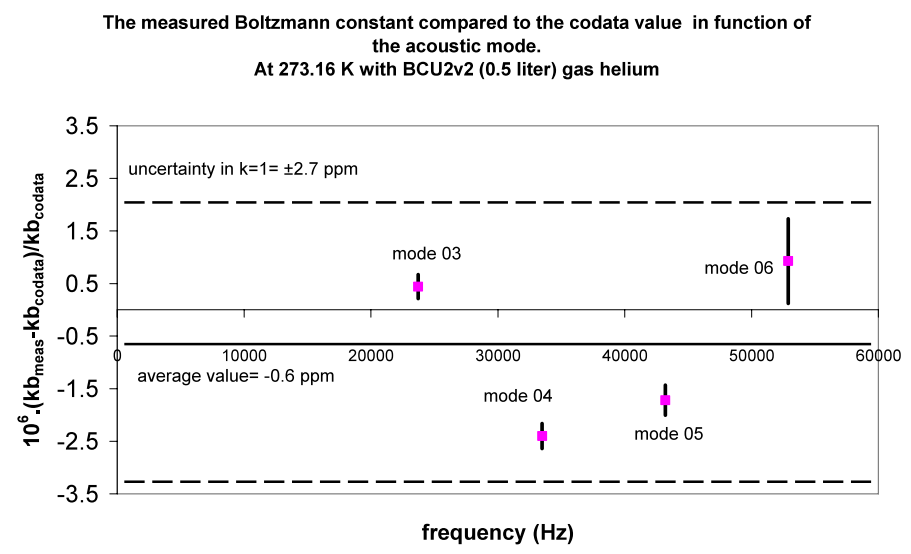

Fig. 12. Relative comparison between the 2008 determinations of the Boltzmann constant $k$ (this work) and the recommended Codata value [23] (in parts in $10^{6}$ ). Experiments have been performed in the BCU2v2 quasi-sphere filled with $0.5 \mathrm{dm}^{3}$ of helium gas at $273.16 \mathrm{~K}$, for the radial modes 03 to 06 . Uncertainty values come from Table 1 .

\section{Conclusion}

This project is entirely devoted to an accurate determination of the Boltzmann constant. It uses methods and techniques belonging to six different areas of physics: temperature measurements, pressure measurements, dimensional measurements, acoustics, thermo-physical properties of gases, electromagnetism. In most of these domains, the expected uncertainty on the parameter to be monitored or measured requires the use of original equipment, at the frontiers of science.

This project will lead to a better understanding of acoustics in our spherical resonator. Once the study is completed, it will lead to a better implementation of the thermodynamic temperature scale on a very wide range of temperatures at LNE-INM/CNAM: $4 \mathrm{~K}$ to $273 \mathrm{~K}$ as a first step, then from $1 \mathrm{~K}$ to $700 \mathrm{~K}$, using an acoustic thermometer as secondary device, before any further change in the definition of the kelvin.

The measurement of the acoustic properties of resonators also provides access to a precise measurement of some of the characteristic parameters of the gas filling the cavity. Experiments have already been made in the past to determine the electrical neutrality of matter [20,21] (a problem in some areas of physics) or the viscosity of gases [22] (needed in some industrial applications, as micro-electronics). Other experimental studies can lead to the determination of other physical properties of the gas, such as relaxation parameters, coefficient of thermal diffusion, etc. The reproducibility of the measurements, the control of the experimental conditions, the availability of a refined model of the acoustic fields and, more generally, the ability to control the measurement procedures and devices (particularly because models are both an aid for the design and optimisation of experiments and for the assessment of uncertainties) are key elements to set the performance of such experiments.

\section{References}

[1] Résolution 3, $10^{\mathrm{e}}$ Conférence générale des poids et mesures, 1954.

[2] Résolution $12,11^{\mathrm{e}}$ Conférence générale des poids et mesures, 1960.

[3] Résolution 7, $21^{\mathrm{e}}$ Conférence générale des poids et mesures, 1999.

[4] Résolution $5,20^{\mathrm{e}}$ Conférence générale des poids et mesures, 1995.

[5] Résolution $1,17^{\mathrm{e}}$ Conférence générale des poids et mesures, 1983.

[6] J.W.S. Rayleigh, Theory of Sound, 2nd ed., Dover, 1945.

[7] P.M. Morse, K.U. Ingard, Theoretical Acoustics, McGraw-Hill, New York, 1968.

[8] M. Bruneau, Manuel d'acoustique fondamentale, Hermès, Paris, 1998.

[9] C. Guianvarc'h, L. Pitre, M. Bruneau, A.M. Bruneau, Acoustic field in a quasi-spherical resonator: Unified perturbation model, J. Acoust. Soc. Am. 123 (2009) 1416-1425.

[10] M.R. Moldover, J.P. Trusler, T.J. Edwards, J.B. Mehl, R.S. Davis, Measurement of the universal gas constant $R$ using a spherical acoustic resonator, J. Res. Natl. Bur. Stand. 93 (1988) 85-144.

[11] M.R. Moldover, J.B. Mehl, M. Greenspan, Gas-filled spherical resonators: Theory and experiment, J. Acoust. Soc. Am. 79 (1986) 253.

[12] W.G. Thomas, M.J. Preslar, J.C. Farmer, Calibration of condenser microphones under increased atmospheric pressures, J. Acoust. Soc. Am. 51 (1972) 6. 
[13] K. Rasmussen, The static pressure and temperature coefficients of laboratory standard microphones, Metrologia 36 (1999) $265-273$.

[14] C. Guianvarc'h, R.M. Gavioso, G. Benedetto, L. Pitre, M. Bruneau, Characterization of condenser microphones under different environmental conditions for accurate speed of sound measurements with acoustic resonators, Rev. Sci. Instrum. 80 (2009) 074901-10.

[15] E.F. May, L. Pitre, J.B. Mehl, M.R. Moldover, J.W. Schmidt, Quasi-spherical cavity resonators for metrology based on the relative dielectric permittivity of gases, Rev. Sci. Instrum. 75, 3307.

[16] L. Pitre, M.R. Moldover, W.L. Tew, Acoustic thermometry: New results from 273 K to 77 K and progress towards 4 K, Metrologia 43 (2006) $142-162$.

[17] F. Sparasci, L. Pitre, Y. Hermier, Realization of the triple point of water in metallic sealed cells at the LNE-INM/CNAM: A Progress Report, in: 10th Tempmeko, Lake Louise, Canada, 21-25 May 2007, Int. J. Thermophys. 29 (3) (2008) 825-835.

[18] J.B. Mehl, Second-order electromagnetic eigenfrequencies of a triaxial ellipsoid, Metrologia 46 (2009) 554-559.

[19] L. Pitre, C. Guianvarc'h, F. Sparasci, A. Richard, D. Truong, Progress towards an acoustic/microwave determination of the Boltzmann constant at LNE-INM/CNAM: Progress Report, in: 10th Tempmeko, Lake Louise, Canada, 21-25 May 2007, Int. J. Thermophys. 29 (5) (2008) 17301739.

[20] H.F. Dylla, J.G. King, Neutrality of molecules by a new method, Phys. Rev. A 7 (1973) 1224-1229.

[21] C.S. Unnikrishnan, G.T. Gillies, The electrical neutrality of atoms and of bulk matter, Metrologia 41 (2004) S125-S135.

[22] K.A. Gillis, J.B. Mehl, M.R. Moldover, Theory of the Greenspan viscometer, J. Acoust. Soc. Am. 114 (2003) 166-173.

[23] P.J. Mohr, B.N. Taylor, CODATA recommended values of the fundamental physical constants: 2002, Rev. Mod. Phys. 77 (2005) 1-108.

[24] R. Underwood, J.B. Mehl, L. Pitre, G.J. Edwards, G. Sutton, M. De Podesta, Meas. Sci. Technol., submitted for publication. 